\title{
SEPARABILITY BY SEMIVALUES MODIFIED FOR GAMES WITH COALITION STRUCTURE*
}

\author{
RAfael Amer ${ }^{1}$ And José Miguel Giménez ${ }^{2}$
}

\begin{abstract}
Two games are inseparable by semivalues if both games obtain the same allocation whatever semivalue is considered. The problem of separability by semivalues reduces to separability from the null game. For four or more players, the vector subspace of games inseparable from the null game by semivalues contains games different to zero-game. Now, for five or more players, the consideration of a priori coalition blocks in the player set allows us to reduce in a significant way the dimension of the vector subspace of games inseparable from the null game. For these subspaces we provide basis formed by games of a particular type.
\end{abstract}

Keywords. Cooperative games, semivalue, semivalue modified for games with coalition structure, separability, multilinear extension.

Mathematics Subject Classification. 91A12, 91A70.

\section{INTRODUCTION}

Probabilistic values as solution concepts for cooperative games were introduced by Weber [11] in 1988. A probabilistic value assigns to each player a weighted sum

Received February 14, 2008. Accepted March 6, 2009.

* Research partially supported by Grant MTM 2006-06064 of the Education and Science Spanish Ministry and the European Regional Development Fund and Grant SGR 2005-00651 of the Catalonia Government (Generalitat de Catalunya).

1 Department of Applied Mathematics II and Industrial and Aeronautic Engineering School of Terrassa, Technical University of Catalonia, Spain. rafel.amer@upc.edu

2 Department of Applied Mathematics III and Engineering School of Manresa, Technical University of Catalonia, Spain. Corresponding author. Mailing address: EPSEM, Avda. Bases de Manresa 61, 08242 Manresa, Spain. jose.miguel.gimenez@upc.edu 
of its marginal contributions to the coalitions, where the weighting coefficients form a probability distribution over the coalitions to which it belongs.

A particular type of probabilistic value is formed by the semivalues that were defined by Dubey, et al. [5] in 1981. In this case the weighting coefficients are independent of the players and they only depend on the coalition size. Semivalues represent a natural generalization of both the Shapley value [10] and the Banzhaf value $[3,7]$.

It is possible to find two cooperative games that obtain the same payoff vector by each semivalue. We say that these games are inseparable by semivalues. By the linearity property of semivalues, we can reduce the problem of separability between games to separability from the null game. The vector subspace of games inseparable from the null game by semivalues is called shared kernel [1] and its dimension is $2^{n}-n^{2}+n-2$, where $n$ denotes the number of players. For spaces of cooperative games with four or more players, the shared kernel contains games different to the zero game

The probabilistic values are a wide family of solutions: given two different games, it is always possible to find a probabilistic value that separates them. The semivalues also form an important family of solutions. We can evaluate its largeness according to its ability to separate games. Two games are separable if their difference does not belong to the shared kernel. The dimension of this subspace would measure the impossibility of separation.

In this paper we consider a priori coalition blocks in the player set. Each coalition block is a group of players which decide to act together with respect to the rest of the players. The coalition blocks can be economic, political or social agents with affinities or common interests. The coalition blocks act as one unit in a first bargaining process. Later, a new bargaining process occurs among the members of each block. Thus, the existence of coalition blocks implies two levels of interaction among the players: first, among the coalition blocks, and second, within each coalition block.

A semivalue modified for games with coalition structure [2] applies the considered semivalue in both levels of interaction - indeed, as we will see later, the induced semivalues corresponding to both levels. - Our purpose is to reduce the dimension of the vector subspace of games inseparable from the null game. For cooperative games with five or more players, the semivalues modified for games with coalition structure manage to reduce in a significant way the dimension of the shared kernel.

In addition, once an a priori ordering is chosen in the player set, the shared kernel is spanned by specific $\{-1,0,1\}$-valued games so-called shuffle games [1]. Now, we will prove that the vector subspace of games inseparable from the null game by modified semivalues is spanned by games introduced here under the name of expanded shuffle games.

The paper is organized as follows. In Section 2 we consider the solution concepts of semivalue and semivalue modified for games with coalition structure. Also, nomenclature and main results for games inseparable by semivalues are described. 
Section 3 shows that shuffle games which are solutions to the problem of separability by semivalues do not have, in general, the same property with respect to separability by modified semivalues. In Section 4 two sufficient conditions for separability by modified semivalues are proposed. Finally, in Section 5 we determine the dimension and a basis for each vector subspace of games inseparable from the null game by modified semivalues.

\section{Preliminaries}

\section{Cooperative games and semivalues}

A cooperative game with transferable utility or TU game is a pair $(N, v)$, where $N$ is a finite set of players, usually $N=\{1,2, \ldots, n\}$, and $v: 2^{N} \rightarrow \mathbb{R}$ is the so-called characteristic function, which assigns to every coalition $S \subseteq N$ a real number $v(S)$, the worth of coalition $S$, and satisfies the natural condition $v(\emptyset)=0$. We denote the set of all cooperative TU games on $N$ by $G_{N}$. For a given set of players $N$, we identify each game $(N, v)$ with its characteristic function $v$.

A solution or a value on $G_{N}$ is a function $\Psi: G_{N} \rightarrow \mathbb{R}^{N}$ which assigns to every game $v$ a vector $\Psi[v]$ with components $\Psi_{i}[v]$ for all $i \in N$ and it represents a method to measure the negotiation strength of the players in the game. The vector space $\mathbb{R}^{N}$ is called the allocation space. Semivalues as solution concepts were introduced and axiomatically characterized by Dubey et al. [5]. Given a game $v \in G_{N}$ and a semivalue $\psi$ on $G_{N}$, the allocation to each player is an average of the marginal contributions to the coalitions to which it belongs,

$$
\psi_{i}[v]=\sum_{S \subseteq N: i \in S} p_{s}^{n}[v(S)-v(S \backslash\{i\})] \quad \forall i \in N,
$$

where $s=|S|$ and $n=|N|$. The weighting coefficients $p_{s}^{n}$ only depend on the coalition size and verify $\sum_{s=1}^{n}\left(\begin{array}{c}n-1 \\ s-1\end{array}\right) p_{s}^{n}=1$ and $p_{s}^{n} \geq 0$ for $1 \leq s \leq n$. We denote the set of all semivalues on $G_{N}$ by $\operatorname{Sem}\left(G_{N}\right)$.

Let $\alpha \in(0,1)$. We call binomial semivalue $\psi_{\alpha}$ to the semivalue whose coefficients are $p_{\alpha, s}^{n}=\alpha^{s-1}(1-\alpha)^{n-s}$ for $s=1, \ldots, n$. Using the convention that $0^{0}=1$, the definition makes sense also for $\alpha=0$ and $\alpha=1$. We respectively get the dictatorial index for $\alpha=0$ and the marginal index for $\alpha=1:\left(\psi_{0}\right)_{i}[v]=v(\{i\}) \forall i \in N$ and $\left(\psi_{1}\right)_{i}[v]=v(N)-v(N \backslash\{i\}) \forall i \in N$. The Banzhaf value [3,7] is the binomial semivalue for $\alpha=1 / 2$.

It is proven [2] that $n$ distinct binomial semivalues form a reference system in $\operatorname{Sem}\left(G_{N}\right)$ : given $n$ distinct numbers $\alpha_{j}$ in $[0,1]$, for each semivalue $\psi \in \operatorname{Sem}\left(G_{N}\right)$ there exists a unique family of coefficients $\lambda_{j}, 1 \leq j \leq n$, such that $\psi=\sum_{j=1}^{n} \lambda_{j} \psi_{\alpha_{j}}$.

The multilinear extension [6] (MLE in the sequel) of a game $v \in G_{N}$ is the function $f_{v}:[0,1]^{n} \rightarrow \mathbb{R}$ defined by

$$
f_{v}\left(x_{1}, x_{2}, \ldots, x_{n}\right)=\sum_{S \subseteq N} \prod_{i \in S} x_{i} \prod_{j \notin S}\left(1-x_{j}\right) v(S) .
$$


As it happens for the Banzhaf value, the allocation by each binomial semivalue can be computed by replacing in the partial derivatives of the MLE the variables by the value $\alpha[2]$ :

$$
\left(\psi_{\alpha}\right)_{i}[v]=\frac{\partial f_{v}}{\partial x_{i}}(\bar{\alpha}) \quad \forall i \in N, \quad \text { where } \quad \bar{\alpha}=(\alpha, \alpha, \ldots, \alpha) .
$$

In addition, the allocation for any semivalue can be computed by means of a product of two matrices:

$$
\psi[v]=B \Lambda,
$$

where the matrix $B$ depends on each reference system of semivalues $B=$ $\left(b_{i j}\right)_{1 \leq i, j \leq n}$ with $b_{i j}=\left(\psi_{\alpha_{j}}\right)_{i}[v]=\frac{\partial f_{v}}{\partial x_{i}}\left(\overline{\alpha_{j}}\right)$ and $\Lambda$ is the column matrix of the coefficients of $\psi$ in this reference system, $\Lambda^{t}=\left(\lambda_{1} \lambda_{2} \cdots \lambda_{n}\right)$ if $\psi=\sum_{j=1}^{n} \lambda_{j} \psi_{\alpha_{j}}$. This way, a $(n \times n)$-matrix summarizes the payments by any semivalue to all players of a given game $v$.

\section{Modified semivalues}

Given a semivalue $\psi \in \operatorname{Sem}\left(G_{N}\right)$ with weighting coefficients $p_{s}^{n}$, the recursively obtained numbers

$$
p_{s}^{m}=p_{s}^{m+1}+p_{s+1}^{m+1} \quad 1 \leq s \leq m<n,
$$

define an induced semivalue $\psi^{m}$ on the space of cooperative games with $m$ players (see, for instance, [4]). Adding the initial semivalue, the family of induced semivalues $\left\{\psi^{m} \in \operatorname{Sem}\left(G_{M}\right) / 1 \leq m \leq n\right\}$ allowed to define the concept of semivalue modified for games with coalition structure [2] following a similar process to that used by Owen to derive the coalition value [8] from the Shapley value or the modified Banzhaf value for games with coalition structure [9] from the Banzhaf value.

We denote a coalition structure in $N$ by $B=\left\{B_{1}, B_{2}, \ldots, B_{m}\right\}$, i.e., a finite partition of $N, \cup_{k=1}^{m} B_{k}=N$ and $B_{k} \cap B_{l}=\emptyset$ for $k \neq l$. Now, $M=\{1, \ldots, m\}$ is the set of classes in $N$ given by the coalition structure $B$. For each $K \subseteq B_{j}$, we define a modified quotient game

$$
u_{B_{j} \mid K}(L)=v\left(\bigcup_{l \in L} B_{l} \backslash K^{\prime}\right) \quad \forall L \subseteq M,
$$

where $K^{\prime}=B_{j} \backslash K$. This is the game played by the partition classes with the exception of $B_{j}$, that is replaced by the subset $K$. Since the game $u_{B_{j} \mid K}$ is defined on a set $M$ with $m$ players $(1 \leq m \leq n)$, we can apply the induced semivalue $\psi^{m}$ :

$$
w_{j}(K)=\left(\psi^{m}\right)_{j}\left[u_{B_{j} \mid K}\right] \quad \forall K \subseteq B_{j} .
$$

The value $w_{j}(K)$ denotes the strategic position of the subset $K \subseteq B_{j}$ if this subset directly negotiates with the other classes as players in the quotient game, according to the semivalue $\psi$, in absence of $K^{\prime}=B_{j} \backslash K$. 
Next, since the game $w_{j}$ is defined on $B_{j}$, a set with $b_{j}=\left|B_{j}\right|$ players $(1 \leq$ $b_{j} \leq n$ ), we can apply the induced semivalue $\psi^{b_{j}}$ and we define the semivalue $\psi$ modified by the coalition structure $B$ as

$$
\psi_{i}[v ; B]=\left(\psi^{b_{j}}\right)_{i}\left[w_{j}\right] \quad \forall i \in B_{j} .
$$

The semivalue $\psi$ has acted twice: among the coalition blocks and within the coalition block to which the player $i$ belongs. An explicit expression for the allocations according to modified semivalues is derived in [2]:

$$
\psi_{i}[v ; B]=\sum_{S \subseteq B_{j} \backslash\{i\}} \sum_{T \subseteq M \backslash\{j\}} p_{s+1}^{b_{j}} p_{t+1}^{m}\left[v\left(\bigcup_{t \in T} B_{t} \cup S \cup\{i\}\right)-v\left(\bigcup_{t \in T} B_{t} \cup S\right)\right] .
$$

For the coalition structures with individual blocks, $B_{i}=\{\{1\},\{2\}, \ldots,\{n\}\}$, and grand coalition, $B_{t}=\{\{1,2, \ldots, n\}\}$, the modified allocations agree with the allocation by the initial semivalue.

Also, the allocations by modified semivalues can be computed by means of a product of matrices, once a reference system of binomial semivalues has been chosen:

$$
\psi_{i}[v ; B]=\Lambda^{t} A(i) \Lambda .
$$

Matrix $\Lambda$ is like in expression (2.3). The elements $a_{p q}(i), 1 \leq p, q \leq n$, of the matrix $A(i)$ can be obtained from the MLE $f_{v}=f_{v}\left(x_{1}, x_{2}, \ldots, x_{n}\right)$ of a game $v$ by means of the following rules:

(i) For each $t \in M, t \neq j$, and each $m \in B_{t}$ replace the variable $x_{m}$ by $y_{t}$. Thus, a new function of the variables $x_{k}, y_{t}$ for $k \in B_{j}$ and $t \in M \backslash\{j\}$ is obtained.

(ii) In the above function, reduce all exponents that appear in $y_{t}$ to 1 , that is, replace $y_{t}^{r}(r>1)$ by $y_{t}$, obtaining another multilinear function $g_{j}\left(x_{k}, y_{t}\right)$, $k \in B_{j}$ and $t \in M \backslash\{j\}$.

(iii) Calculate the partial derivative of $g_{j}$ with respect to the variable $x_{i}$.

(iv) Replace each $x_{k}$ with $\alpha_{p}$ and each $y_{t}$ with $\alpha_{q}$. Then,

$$
a_{p q}(i)=\frac{\partial g_{j}}{\partial x_{i}}\left(\overline{\alpha_{p}}, \overline{\alpha_{q}}\right) \quad \text { for } \quad 1 \leq p, q \leq n
$$

The function $g_{j}$ is a multilinear function related with the game $v$ and the coalition block $B_{j}$. It is valid for all players in the coalition block $B_{j}$.

One can find a detailed proof and several examples of this procedure to compute allocations by modified semivalues in [2].

\section{Separability by semivalues}

We say that two games $v, v^{\prime} \in G_{N}$ such that $v \neq v^{\prime}$ are separable by a solution $\Psi$ on $G_{N}$ if $\Psi[v] \neq \Psi\left[v^{\prime}\right]$. Since semivalues verify linearity, we only consider separability from the null game. 
For each $G_{N}$, the linear subspace of games inseparable by semivalues from the null game is called shared kernel $C_{N}$. It is proven [1] that games $v \in C_{N}$ verify

$$
\sum_{S: i \in S,|S|=s} v(S)=0 \quad \text { for all } i \in N \text { and } 1 \leq s \leq n .
$$

Grouping these conditions according to coalition sizes, the freedom degrees for each $s$ with $2 \leq s \leq n-2$ are $\left(\begin{array}{l}n \\ s\end{array}\right)-n$, whereas $v(S)=0$ for $|S|=1, n-1, n$. This way, the dimension of $C_{N}$ is $2^{n}-n^{2}+n-2$ for $|N|=n \geq 2$ and $C_{N}=\{0\}$ if $|N|=2,3$.

In game spaces $G_{N}$ with cardinality $|N| \geq 4$, for a given coalition $S \subseteq N$ and players $i, j \in S$ and $k, l \in N \backslash S$, we define the shuffle game $v_{S, i, j, k, l}$ as

$$
v_{S, i, j, k, l}=1_{S}+1_{S \cup\{k, l\} \backslash\{i, j\}}-1_{S \cup\{k\} \backslash\{i\}}-1_{S \cup\{l\} \backslash\{j\}},
$$

where $1_{S}$ is the unity game in $G_{N}\left(1_{S}(S)=1\right.$ and $1_{S}(T)=0$ otherwise). If $v \in G_{N}$ is a shuffle game, then $v \in C_{N}$. In [1], it is proven that the shared kernel is spanned by shuffle games. Since each shuffle game takes non null values uniquely on coalitions of a single size, the number of selected games in the proof of this property is $\left(\begin{array}{l}n \\ s\end{array}\right)-n$ for coalitions $S$ with $2 \leq s \leq n-2(|S|=s)$.

\section{Shuffle Games And COAlition structures}

Proposition 3.1. Let $f_{v}=f_{v}\left(x_{1}, x_{2}, \ldots, x_{n}\right)$ be the MLE of a game $v \in G_{N}$.

$$
v \in C_{N} \Leftrightarrow \nabla f_{v}(\bar{\alpha})=0 \quad \forall \alpha \in[0,1], \quad \bar{\alpha}=(\alpha, \alpha, \ldots, \alpha) .
$$

Proof. If $v \in C_{N}$, then $\psi[v]=0 \forall \psi \in \operatorname{Sem}\left(G_{N}\right)$. In particular, for every binomial semivalue $\psi_{\alpha}$ with $\alpha \in[0,1], \psi_{\alpha}[v]=\nabla f_{v}(\bar{\alpha})=0$ where $\bar{\alpha}=(\alpha, \ldots, \alpha)$.

Conversely, since $n$ binomial semivalues form a reference system in $\operatorname{Sem}\left(G_{N}\right)$, every semivalue $\psi \in \operatorname{Sem}\left(G_{N}\right)$ can uniquely be written like $\psi=\sum_{j=1}^{n} \lambda_{j} \psi_{\alpha_{j}}$ with $\alpha_{j} \in[0,1]$ for $1 \leq j \leq n$. Then,

$$
\psi[v]=\sum_{j=1}^{n} \lambda_{j} \psi_{\alpha_{j}}[v]=\sum_{j=1}^{n} \lambda_{j} \nabla f_{v}\left(\overline{\alpha_{j}}\right)=0
$$

and the game $v$ belongs to the shared kernel $C_{N}$.

Example 3.2. Let $N=\{i, j, k, l\}$ be the set of players. For cooperative games with four players the coalition $S$ in the shuffle games is only composed by two players. For short, when $S=\{i, j\}$ we write the shuffle game $v_{S, i, j, k, l}$ as $v_{i, j, k, l}$, i.e.,

$$
v_{i, j, k, l}=1_{\{i, j\}}+1_{\{k, l\}}-1_{\{j, k\}}-1_{\{i, l\}} .
$$

The MLE of this game is $f_{v_{i, j, k}}=x_{i} x_{j}+x_{k} x_{l}-x_{j} x_{k}-x_{i} x_{l}$.

It is easy to see that $\nabla f_{v_{i, j, k},}(\bar{\alpha})=0 \forall \alpha \in[0,1]$, as one expects for a game inseparable from the null game by semivalues. 
Definition 3.3. We say that a cooperative game $v \in G_{N}$ is inseparable from the null game by semivalues modified for games with coalition structure if and only if $\psi[v ; B]=0$ for every semivalue $\psi$ on $G_{N}$ and every coalition structure $B$ in $N$.

The above definition introduces our central concept of separability between games by modified semivalues. According to the linearity property, the concept reduces to separability from the null game. Now, shuffle games that give the solution to the problem of separability by semivalues, offer a different answer according to the cardinality of the player set.

Proposition 3.4. Let $G_{N}$ be the vector space of cooperative TU games with four players, $|N|=4$. A game $v \in G_{N}$ is inseparable from the null game by semivalues if and only if it is inseparable from the null game by semivalues modified for games with coalition structure.

Proof. For case $|N|=4$, the shared kernel $C_{N}$ has dimension 2. According to the development in [1], a basis for $C_{N}$ is formed by the shuffle games $v_{1,4,3,2}$ and $v_{2,4,3,1}$. For the shuffle games in a basis of $C_{N}$, we will prove that the condition of inseparability from the null game by semivalues extends to the condition of inseparability from the null game by modified semivalues. For the remaining games in $C_{N}$, the property is verified by linearity.

We consider, for example, game $v_{2,4,3,1}$ and simultaneously all possible types of coalition structures in $N=\{1,2,3,4\}$. (a) Four individual blocks. (b) One bipersonal block where game $v_{2,4,3,1}$ takes non-null value and two individual blocks. (c) Like in (b) but taking null value. (d) Two bipersonal blocks where game $v_{2,4,3,1}$ takes non-null values. (e) Like in (d) but taking null values. (f) One coalition block with three players. (g) Only one coalition block with four players.

In cases (a) and (g), both allocations coincide: $\psi\left[v_{2,4,3,1} ; B\right]=\psi\left[v_{2,4,3,1}\right]=0$ $\forall \psi \in \operatorname{Sem}\left(G_{N}\right), B=\{\{1\},\{2\},\{3\},\{4\}\}$ or $B=\{\{1,2,3,4\}\}$.

From now on we will use the MLE $f_{v_{2,4,3,1}}=x_{2} x_{4}+x_{1} x_{3}-x_{3} x_{4}-x_{1} x_{2}$.

Case (b). We consider, for instance, the coalition structure $B=\{\{1,2\},\{3\},\{4\}\}$. According to the rules that lead to the terms in expression (2.6) for obtaining the value $\psi_{1}\left[v_{2,4,3,1} ; B\right]$ by means of a product of matrices as in $(2.5)$, we first determine the modified MLE $g_{1}$ :

$$
\begin{gathered}
g_{1}\left(x_{1}, x_{2}, y_{2}, y_{3}\right)=x_{2} y_{3}+x_{1} y_{2}-y_{2} y_{3}-x_{1} x_{2} ; \\
\frac{\partial g_{1}}{\partial x_{1}}=y_{2}-x_{2} \Rightarrow a_{p q}(1)=\frac{\partial g_{1}}{\partial x_{1}}\left(\overline{\alpha_{p}}, \overline{\alpha_{q}}\right)=\alpha_{q}-\alpha_{p} \quad \text { for } 1 \leq p, q \leq 4 .
\end{gathered}
$$

Written any semivalue $\psi$ as a linear combination of four different binomial semivalues, we can conclude that

$$
\psi_{1}\left[v_{2,4,3,1} ; B\right]=\Lambda^{t} A(1) \Lambda=0 \quad \forall \psi \in \operatorname{Sem}\left(G_{N}\right),
$$

since, in this case, matrix $A(1)$ satisfies $a_{p q}(1)=-a_{q p}(1)$ for $1 \leq p, q \leq 4$. In a similar way, $\psi_{2}\left[v_{2,4,3,1} ; B\right]=0 \forall \psi \in \operatorname{Sem}\left(G_{N}\right)$. 
Now, for obtaining the value $\psi_{3}\left[v_{2,4,3,1} ; B\right]$, we determine the modified MLE $g_{2}$ :

$$
\begin{gathered}
g_{2}\left(y_{1}, x_{3}, y_{3}\right)=y_{1} y_{3}+y_{1} x_{3}-x_{3} y_{3}-y_{1} ; \\
\frac{\partial g_{2}}{\partial x_{3}}=y_{1}-y_{3} \Rightarrow a_{p q}(3)=\frac{\partial g_{2}}{\partial x_{3}}\left(\overline{\alpha_{p}}, \overline{\alpha_{q}}\right)=0 \quad \text { for } 1 \leq p, q \leq 4 .
\end{gathered}
$$

Then $\psi_{3}\left[v_{2,4,3,1} ; B\right]=0$ and, also, $\psi_{4}\left[v_{2,4,3,1} ; B\right]=0$.

Case (c). Possible coalition structure $B=\{\{1,4\},\{2\},\{3\}\}$.

$$
\begin{gathered}
g_{1}\left(x_{1}, x_{4}, y_{2}, y_{3}\right)=y_{2} x_{4}+x_{1} y_{3}-y_{3} x_{4}-x_{1} y_{2} ; \\
\frac{\partial g_{1}}{\partial x_{1}}=y_{3}-y_{2} \Rightarrow a_{p q}(1)=\frac{\partial g_{1}}{\partial x_{1}}\left(\overline{\alpha_{p}}, \overline{\alpha_{q}}\right)=0 \quad \text { for } 1 \leq p, q \leq 4 .
\end{gathered}
$$

Consequently, $\psi_{1}\left[v_{2,4,3,1} ; B\right]=0$. In a similar way, $\psi_{4}\left[v_{2,4,3,1} ; B\right]=0$ and $\psi_{2}\left[v_{2,4,3,1} ; B\right]=\psi_{3}\left[v_{2,4,3,1} ; B\right]=0$.

Similar manipulations of the MLE $f_{v_{2,4,3,1}}$ in cases (d), (e) and (f) give rise to the same conclusion: $\psi\left[v_{2,4,3,1} ; B\right]=0$.

Conversely, if a game is inseparable from the null game by modified semivalues, in particular, it is inseparable from the null game by semivalues. It suffices to consider the coalition structure formed by individual blocks.

Proposition 3.5. Let $G_{N}$ a vector space of cooperative TU games with five or more players. Every shuffle game in $G_{N}$ is separable from the null game by semivalues modified for games with coalition structure.

Proof. In $G_{N}$ with $|N| \geq 5$, the shuffle game $v_{S, i, j, k, l}=1_{S}+1_{S \cup\{k, l\} \backslash\{i, j\}}-$ $1_{S \cup\{k\} \backslash\{i\}}-1_{S \cup\{l\} \backslash\{j\}}$, with $i, j \in S$ and $k, l \in N \backslash S$, has by MLE

$$
f_{v_{S, i, j, k, l}}=\left[x_{i} x_{j}+x_{k} x_{l}-x_{j} x_{k}-x_{i} x_{l}\right] \prod_{p \in S \backslash\{i, j\}} x_{p} \prod_{q \in N \backslash(S \cup\{k, l\})}\left(1-x_{q}\right) .
$$

For coalitions $S$ with $2 \leq|S|<n-2$, we consider the coalition structure $B_{S}=$ $\{S, N \backslash S\}$. The modified MLE $g_{1}$ for players in block $S$ is

$$
g_{1}=x_{i} x_{j}\left(1-y_{2}\right) \prod_{p \in S \backslash\{i, j\}} x_{p} \quad \text { and } \quad \frac{\partial g_{1}}{\partial x_{i}}=x_{j}\left(1-y_{2}\right) \prod_{p \in S \backslash\{i, j\}} x_{p},
$$

where $N \backslash(S \cup\{k, l\}) \neq \emptyset$ since $|S|<n-2$.

Then, the modified Banzhaf value $\beta$ separates the game $v_{S, i, j, k, l}, 2 \leq|S|<n-2$, from the null game:

$$
\beta_{i}\left[v_{S, i, j, k, l} ; B_{S}\right]=\frac{\partial g_{1}}{\partial x_{i}}(\overline{1 / 2}, \overline{1 / 2})=\frac{1}{2^{s}} \neq 0 .
$$

For case $|S|=n-2, S=N \backslash\{k, l\}$ and the MLE is

$$
f_{v_{N \backslash\{k, l\}, i, j, k, l}}=\left[x_{i} x_{j}+x_{k} x_{l}-x_{j} x_{k}-x_{i} x_{l}\right] \prod_{p \in N \backslash\{i, j, k, l\}} x_{p} .
$$


Now, we consider the coalition structure $B_{N \backslash\{k, l\}}=\{N \backslash\{k, l\},\{k, l\}\}$ and we obtain the modified MLE $g_{1}$ for players in block $N \backslash\{k, l\}$ :

$$
g_{1}=\left[x_{i} x_{j}+y_{2}-x_{j} y_{2}-x_{i} y_{2}\right] \prod_{p \in N \backslash\{i, j, k, l\}} x_{p},
$$

where $N \backslash\{i, j, k, l\} \neq \emptyset$ since $|N| \geq 5$. Let $h$ be a player in $N \backslash\{i, j, k, l\}$. Again, the modified Banzhaf value $\beta$ separates the game $v_{N \backslash\{k, l\}, i, j, k, l}$ from the null game:

$$
\frac{\partial g_{1}}{\partial x_{h}}=\left[x_{i} x_{j}+y_{2}-x_{j} y_{2}-x_{i} y_{2}\right] \prod_{p \in N \backslash\{h, i, j, k, l\}} x_{p}
$$

and

$$
\beta_{h}\left[v_{N \backslash\{k, l\}, i, j, k, l} ; B_{N \backslash\{k, l\}}\right]=\frac{\partial g_{1}}{\partial x_{h}}(\overline{1 / 2}, \overline{1 / 2})=\frac{1}{2^{n-3}} \neq 0 .
$$

\section{Sufficient CONDitions OF SEPARABILITY}

In the previous section we have seen that, for games with five or more players, the shuffle games do not solve the problem of inseparability by semivalues modified for games with coalition structure. In this section we provide two sufficient conditions of separability, that is, two necessary conditions of inseparability from the null game by modified semivalues.

The inseparability from the null game by semivalues demands a certain condition of complementarity for the coalitions of extreme sizes: $v(N)=v(\emptyset)=0$ and $v(N \backslash\{i\})=v(\{i\})=0$ for all $i \in N$ (see (2.7) and subsequent comment). Proposition 4.1 will show that this condition of complementarity must extend to all coalitions $S$ and $N \backslash S$ in order to assure the inseparability by modified semivalues.

Later, once the first necessary condition is assumed $(v(S)=v(N \backslash S) \forall S \subseteq N)$, Proposition 4.2 will introduce a more elaborated requirement: the utility of each coalition must reduce to the utility of all contained bipersonal coalitions. The bipersonal coalitions are the most elementary coalition blocks, since in our context, the unipersonal coalitions obtain null utility. A game with a coalition whose utility is not the sum of utilities of its contained bipersonal coalitions can be separated from the null game by a certain coalition structure and a certain semivalue.

Proposition 4.1. Let $G_{N}$ be a vector space of cooperative TU games with four or more players, $|N| \geq 4$, and let $v$ be a game in $G_{N}$. If there exists a coalition $S$ with $v(S) \neq v(N \backslash S)$, then the game $v$ is separable from the null game by semivalues modified for games with coalition structure.

Proof. Let us suppose $S^{\prime}$ a coalition with smallest size that verifies $v\left(S^{\prime}\right) \neq v(N \backslash$ $\left.S^{\prime}\right)$. If $\left|S^{\prime}\right|=1$, the game $v$ is separable from the null game by semivalues and also by modified semivalues. We can consider that $\left|S^{\prime}\right|=s^{\prime} \geq 2$ and $s^{\prime} \leq n / 2$. Then, 
the MLE of the game $v$ can be written as

$$
\begin{aligned}
f_{v}= & \sum_{S: 2 \leq|S| \leq s^{\prime}}\left[\prod_{i \in S} x_{i} \prod_{j \in N \backslash S}\left(1-x_{j}\right) v(S)+\prod_{i \in N \backslash S} x_{i} \prod_{j \in S}\left(1-x_{j}\right) v(N \backslash S)\right] \\
& +\sum_{S: s^{\prime}<|S|<n-s^{\prime}} \prod_{i \in S} x_{i} \prod_{j \in N \backslash S}\left(1-x_{j}\right) v(S) .
\end{aligned}
$$

Now, we choose the coalition structure $B_{S^{\prime}}=\left\{S^{\prime}, N \backslash S^{\prime}\right\}$. In such a case, the modified MLE $g_{1}$ for players in coalition block $S^{\prime}$ has by expression

$$
\begin{aligned}
g_{1}= & \sum_{S \subset S^{\prime}, s \geq 2}\left[\left(1-y_{2}\right) \prod_{i \in S} x_{i} \prod_{j \in S^{\prime} \backslash S}\left(1-x_{j}\right)+y_{2} \prod_{i \in S^{\prime} \backslash S} x_{i} \prod_{j \in S}\left(1-x_{j}\right)\right] v(S) \\
& +\left(1-y_{2}\right) \prod_{i \in S^{\prime}} x_{i} v\left(S^{\prime}\right)+y_{2} \prod_{j \in S^{\prime}}\left(1-x_{j}\right) v\left(N \backslash S^{\prime}\right),
\end{aligned}
$$

because the terms for coalitions $S$ containing elements as well in $S^{\prime}$ as in $N \backslash S^{\prime}$ vanish in the MLE $g_{1}$. If $k$ is a player in $S^{\prime}$,

$$
\begin{aligned}
\frac{\partial g_{1}}{\partial x_{k}}= & \sum_{S \subset S^{\prime}, s \geq 2, S \ni k}\left[\left(1-y_{2}\right) \prod_{i \in S \backslash\{k\}} x_{i} \prod_{j \in S^{\prime} \backslash S}\left(1-x_{j}\right)\right. \\
& \left.-y_{2} \prod_{i \in S^{\prime} \backslash S} x_{i} \prod_{j \in S \backslash\{k\}}\left(1-x_{j}\right)\right] v(S) \\
& +\sum_{S \subset S^{\prime}, s \geq 2, S \ngtr k}\left[-\left(1-y_{2}\right) \prod_{i \in S} x_{i} \prod_{j \in S^{\prime} \backslash(S \cup\{k\})}\left(1-x_{j}\right)\right. \\
& \left.+y_{2} \prod_{i \in S^{\prime} \backslash(S \cup\{k\})} x_{i} \prod_{j \in S}\left(1-x_{j}\right)\right] v(S) \\
& +\left(1-y_{2}\right) \prod_{i \in S^{\prime} \backslash\{k\}} x_{i} v\left(S^{\prime}\right)-y_{2} \prod_{j \in S^{\prime} \backslash\{k\}}\left(1-x_{j}\right) v\left(N \backslash S^{\prime}\right) .
\end{aligned}
$$

Then

$$
\frac{\partial g_{1}}{\partial x_{k}}(\overline{1 / 2}, \overline{1 / 2})=\frac{1}{2^{s^{\prime}}}\left[v\left(S^{\prime}\right)-v\left(N \backslash S^{\prime}\right)\right]
$$

and the modified Banzhaf value $\beta$ separates the game $v$ from the null game:

$$
\beta_{k}\left[v ; B_{S^{\prime}}\right]=\frac{\partial g_{1}}{\partial x_{k}}(\overline{1 / 2}, \overline{1 / 2}) \neq 0 \quad \text { for } k \in S^{\prime}
$$


Proposition 4.2. Let $G_{N}$ be a vector space of cooperative TU games with six or more players, $|N| \geq 6$, and let $v$ be a game in $G_{N}$ that satisfies $v(S)=v(N \backslash S)$ $\forall S \subseteq N$ and $v(\{i\})=0 \forall i \in N$. If there exists a coalition $S$ such that

$$
v(S) \neq \sum_{T \subset S,|T|=2} v(T) \quad \text { and } \quad 3 \leq|S| \leq n / 2,
$$

then the game $v$ is separable from the null game by semivalues modified for games with coalition structure.

Proof. The MLE of a game $v$ that satisfies the two first conditions of the statement can be written as

$$
\begin{aligned}
f_{v}= & \sum_{S: 2 \leq|S|<n / 2}\left[\prod_{i \in S} x_{i} \prod_{j \in N \backslash S}\left(1-x_{j}\right)+\prod_{i \in N \backslash S} x_{i} \prod_{j \in S}\left(1-x_{j}\right)\right] v(S) \\
& +\sum_{S:|S|=n / 2} \prod_{i \in S} x_{i} \prod_{j \in N \backslash S}\left(1-x_{j}\right) v(S),
\end{aligned}
$$

where the second sum only appears in case $n$ even number. Let us suppose $S^{\prime}$ a coalition with smallest size that verifies (4.1) for $\left|S^{\prime}\right|<n / 2$. In such a case, we choose the coalition structure $B_{S^{\prime}}=\left\{S^{\prime}, N \backslash S^{\prime}\right\}$ and we write the modified MLE $g_{1}$ for the players in the coalition block $S^{\prime}$ :

$$
\begin{aligned}
g_{1}= & \sum_{S \subset S^{\prime}, 2 \leq s<s^{\prime}}\left[\left(1-y_{2}\right) \prod_{i \in S} x_{i} \prod_{j \in S^{\prime} \backslash S}\left(1-x_{j}\right)+y_{2} \prod_{i \in S^{\prime} \backslash S} x_{i} \prod_{j \in S}\left(1-x_{j}\right)\right] v(S) \\
& +\left[\left(1-y_{2}\right) \prod_{i \in S^{\prime}} x_{i}+y_{2} \prod_{j \in S^{\prime}}\left(1-x_{j}\right)\right] v\left(S^{\prime}\right) .
\end{aligned}
$$

Next, we consider a certain player $j_{1}$ in the block $S^{\prime}$, we compute the partial derivative of the MLE $g_{1}$ with respect to the variable $x_{j_{1}}$ and we replace all variables by the generic value $\alpha$ by grouping the sums as follows:

$$
\begin{aligned}
\frac{\partial g_{1}}{\partial x_{j_{1}}}(\bar{\alpha}, \bar{\alpha})= & \sum_{S \subset S^{\prime}, S \ni j_{1},|S|=2}\left[\alpha(1-\alpha)^{s^{\prime}-1}-\alpha^{s^{\prime}-1}(1-\alpha)\right] v(S) \\
& +\sum_{S \subset S^{\prime}, S \ni j_{1}, 2<s<s^{\prime}}\left[\alpha^{s-1}(1-\alpha)^{s^{\prime}-s+1}-\alpha^{s^{\prime}-s+1}(1-\alpha)^{s-1}\right] v(S) \\
& +\sum_{S \subset S^{\prime}, S \not \supset j_{1}, 2 \leq s<s^{\prime}-1}\left[\alpha^{s^{\prime}-s}(1-\alpha)^{s}-\alpha^{s}(1-\alpha)^{s^{\prime}-s}\right] v(S) \\
& +\left[\alpha(1-\alpha)^{s^{\prime}-1}-\alpha^{s^{\prime}-1}(1-\alpha)\right]\left[v\left(S^{\prime} \backslash\left\{j_{1}\right\}\right)-v\left(S^{\prime}\right)\right]
\end{aligned}
$$


All terms for coalitions $S$ with $S \not \supset j_{1}$ and $2 \leq s<s^{\prime}-1$ can be written by means of coalitions $T$ with $T \ni j_{1}$ and $3 \leq t<s^{\prime}$. Then,

$$
\begin{aligned}
\frac{\partial g_{1}}{\partial x_{j_{1}}}(\bar{\alpha}, \bar{\alpha}) & =\alpha(1-\alpha)\left[(1-\alpha)^{s^{\prime}-2}-\alpha^{s^{\prime}-2}\right] \\
& \times\left\{\sum_{S \subset S^{\prime}, S \ni j_{1},|S|=2} v(S)+v\left(S^{\prime} \backslash\left\{j_{1}\right\}\right)-v\left(S^{\prime}\right)\right\} \\
& +\sum_{S \subset S^{\prime}, S \ni j_{1}, 2<s<s^{\prime}}\left[\alpha^{s-1}(1-\alpha)^{s^{\prime}-s+1}-\alpha^{s^{\prime}-s+1}(1-\alpha)^{s-1}\right] v(S) \\
& +\sum_{T \subset S^{\prime}, T \ni j_{1}, 2<t<s^{\prime}}\left[\alpha^{s^{\prime}-t+1}(1-\alpha)^{t-1}-\alpha^{t-1}(1-\alpha)^{s^{\prime}-t+1}\right] v\left(T \backslash\left\{j_{1}\right\}\right) .
\end{aligned}
$$

We shorten the polynomial $(1-\alpha)^{s^{\prime}-2}-\alpha^{s^{\prime}-2}$ by means of $p_{s^{\prime}}(\alpha)$ and we write $v\left(S^{\prime} \backslash\left\{j_{1}\right\}\right)$ as a sum of utilities of contained bipersonal coalitions:

$$
\begin{aligned}
& \frac{\partial g_{1}}{\partial x_{j_{1}}}(\bar{\alpha}, \bar{\alpha})=\alpha(1-\alpha) p_{s^{\prime}}(\alpha)\left[\sum_{S \subset S^{\prime}, S \ni j_{1},|S|=2} v(S)+\sum_{T \subseteq S^{\prime} \backslash\left\{j_{1}\right\},|T|=2} v(T)-v\left(S^{\prime}\right)\right] \\
& +\sum_{S \subset S^{\prime}, S \ni j_{1}, 2<s<s^{\prime}}\left[\alpha^{s-1}(1-\alpha)^{s^{\prime}-s+1}-\alpha^{s^{\prime}-s+1}(1-\alpha)^{s-1}\right]\left[v(S)-v\left(S \backslash\left\{j_{1}\right\}\right)\right] .
\end{aligned}
$$

It is possible to find coalitions $S$ with $S \subset S^{\prime}, S \ni j_{1}$ and $2<s<s^{\prime}$ only in case $s^{\prime} \geq 4$. Then, the last sum in the above expression can be written as

$$
\begin{aligned}
& \sum_{S \subset S^{\prime}, S \ni j_{1}, 3 \leq s<1+s^{\prime} / 2}\left[\alpha^{s-1}(1-\alpha)^{s^{\prime}-s+1}-\alpha^{s^{\prime}-s+1}(1-\alpha)^{s-1}\right]\left[v(S)-v\left(S \backslash\left\{j_{1}\right\}\right)\right] \\
& \quad+\sum_{T \subset S^{\prime}, T \ni j_{1}, 1+s^{\prime} / 2<t \leq s^{\prime}-1}\left[\alpha^{t-1}(1-\alpha)^{s^{\prime}-t+1}-\alpha^{s^{\prime}-t+1}(1-\alpha)^{t-1}\right]\left[v(T)-v\left(T \backslash\left\{j_{1}\right\}\right)\right],
\end{aligned}
$$

where case $s=1+s^{\prime} / 2$ is not considered, since the size of $S$ only can take value $s=1+s^{\prime} / 2$ in case $s^{\prime}$ even number but, in this case, the coefficient $\alpha^{s-1}(1-$ $\alpha)^{s^{\prime}-s+1}-\alpha^{s^{\prime}-s+1}(1-\alpha)^{s-1}$ is null. In the above sums, we can identify coalitions $S$ for $3 \leq s<1+s^{\prime} / 2$ with coalitions $T$ for $1+s^{\prime} / 2<t \leq s^{\prime}-1$ by means of $t=s^{\prime}-s+2$. Then, both sums reduce to

$$
\begin{aligned}
& \sum_{3 \leq s<1+s^{\prime} / 2}\left[\alpha^{s-1}(1-\alpha)^{s^{\prime}-s+1}-\alpha^{s^{\prime}-s+1}(1-\alpha)^{s-1}\right] \\
& \times\left\{\sum_{S \subset S^{\prime}, S \ni j_{1},|S|=s}\left[v(S)-v\left(S \backslash\left\{j_{1}\right\}\right)\right]-\sum_{T \subset S^{\prime}, T \ni j_{1},|T|=s^{\prime}-s+2}\left[v(T)-v\left(T \backslash\left\{j_{1}\right\}\right)\right]\right\} .
\end{aligned}
$$


Let us suppose $S^{\prime}=\left\{j_{1}, j_{2}, \ldots, j_{s^{\prime}}\right\}$. For a given cardinality $s$ with $3 \leq s<1+s^{\prime} / 2$, the last difference of sums vanish, because it can be written as

$$
\begin{aligned}
\sum_{S \subset S^{\prime}, S \ni j_{1},|S|=s} & {\left[\sum_{P \subset S,|P|=2} v(P)-\sum_{Q \subseteq S \backslash\left\{j_{1}\right\},|Q|=2} v(Q)\right] } \\
& -\sum_{T \subset S^{\prime}, T \ni j_{1},|T|=s^{\prime}-s+2}\left[\sum_{P \subset T,|P|=2} v(P)-\sum_{Q \subset T \backslash\left\{j_{1}\right\},|Q|=2} v(Q)\right]= \\
\sum_{S \subset S^{\prime}, S \ni j_{1},|S|=s} & {\left[\sum_{P \subset S, P \ni j_{1},|P|=2} v(P)\right]-\sum_{T \subset S^{\prime}, T \ni j_{1},|T|=s^{\prime}-s+2}\left[\sum_{P \subset T, P \ni j_{1},|P|=2} v(P)\right] } \\
& =\sum_{i=2}^{s^{\prime}}\left[\left(\begin{array}{c}
s^{\prime}-2 \\
s-2
\end{array}\right)-\left(\begin{array}{c}
s^{\prime}-2 \\
s^{\prime}-s
\end{array}\right)\right] v\left(\left\{j_{1}, j_{i}\right\}\right)=0 .
\end{aligned}
$$

Thus, from expression (4.3), we can write the modified binomial semivalue $\psi_{\alpha}$ for a certain player $j_{1} \in S^{\prime}$ as

$$
\left(\psi_{\alpha}\right)_{j_{1}}\left[v ; B_{S^{\prime}}\right]=\frac{\partial g_{1}}{\partial x_{j_{1}}}(\bar{\alpha}, \bar{\alpha})=\alpha(1-\alpha) p_{s^{\prime}}(\alpha)\left[\sum_{T \subset S^{\prime},|T|=2} v(T)-v\left(S^{\prime}\right)\right] .
$$

Since $\alpha=1 / 2$ is the unique real zero of the polynomial $p_{s^{\prime}}$ for values $s^{\prime} \geq 3$ and the game $v$ satisfies the inequality (4.1) for the coalition $S^{\prime}$, we conclude that $\left(\psi_{\alpha}\right)_{j_{1}}\left[v ; B_{S^{\prime}}\right] \neq 0$ for values $\alpha \in(0,1 / 2) \cup(1 / 2,1)$ and these modified semivalues separate the game $v$ from the null game.

Finally, we have to see the case $|S|=n / 2$, when $n / 2$ is the smallest size of the coalitions that verify condition (4.1) in the statement. Here, $n$ is an even number and all coalitions in the second sum of expression (4.2), for $|S|=n / 2$, can be grouped by pairs: $S$ and $N \backslash S$ with $v(N \backslash S)=v(S)$. Now, the selected coalition for the procedure $S^{\prime}$ will belong to one or another half of the coalitions with size $n / 2$; we choose half that contains coalition $S^{\prime}$ and describe the second sum with $S$ as the same way that the first sum in expression (4.2). Then, by repeating the same procedure as in case $|S|<n / 2$, we reach the same conclusion.

\section{EXPANDED SHUfFle GAMES}

In this section we define a particular type of games related to the shuffle games that verify both necessary conditions of inseparability introduced in Propositions 4.1 and 4.2. We denote by $D_{N}$ the vector subspace of all cooperative games in $G_{N}$ inseparable from the null game by semivalues modified for games with coalition structure. 
Definition 5.1. In $G_{N}$ with $|N| \geq 5$ we consider a shuffle game with coalition size $2, v_{i, j, k, l}, k, l \in N \backslash\{i, j\}$. The expanded shuffle game $v_{i, j, k, l}^{e}$ related to the shuffle game $v_{i, j, k, l}$ is the sum of all shuffle games in $G_{N}, v_{P, i, j, k, l}$, with the same shuffled players, i.e.,

$$
v_{i, j, k, l}^{e}=\sum_{P \ni i, j, P \subseteq N \backslash\{k, l\}} v_{P, i, j, k, l} .
$$

Lemma 5.2. In $G_{N}$ with $|N| \geq 5$ an expanded shuffle game $v_{i, j, k, l}^{e}, k, l \in N \backslash$ $\{i, j\}$, satisfies the following properties:

(a) $v_{i, j, k, l}^{e}(S)=v_{i, j, k, l}^{e}(N \backslash S) \quad \forall S \subseteq N$;

(b) $v_{i, j, k, l}^{e}(S)=\sum_{T \subset S,|T|=2} v_{i, j, k, l}^{e}(T) \quad \forall S \subseteq N$ and $3 \leq|S| \leq|N|$;

(c) its MLE is $f_{v_{i, j, k, l}^{e}}=x_{i} x_{j}+x_{k} x_{l}-x_{j} x_{k}-x_{i} x_{l}$.

Proof. It is easy to prove sections (a) and (b); it suffices to check if the players $i, j, k, l$ belong or not to each coalition $S$, since the unique bipersonal coalitions that take non-null values in game $v_{i, j, k, l}^{e}$ are $\{i, j\},\{k, l\},\{j, k\}$ and $\{i, l\}$. In order to verify section (c) we can write the MLE of the game $v_{i, j, k, l}^{e}$ as

$$
f_{v_{i, j, k, l}^{e}}=\left[x_{i} x_{j}+x_{k} x_{l}-x_{j} x_{k}-x_{i} x_{l}\right]\left[\prod_{q \in N \backslash\{i, j, k, l\}}\left(1-x_{q}\right)+f_{\left.\sum_{Q \subseteq N \backslash\{i, j, k, l\}} 1_{Q}\right],}\right.
$$

where the games $1_{Q}$ are considered in $G_{N \backslash\{i, j, k, l\}}$. Since $\sum_{Q \subseteq N \backslash\{i, j, k, l\}} 1_{Q}(T)=1$ $\forall T \subseteq N \backslash\{i, j, k, l\}, T \neq \emptyset,(Q \neq \emptyset)$, its MLE equals the unity in $N \backslash\{i, j, k, l\}$ and section (c) follows.

Proposition 5.3. In spaces of cooperative TU games $G_{N}$ with $|N| \geq 5$ every expanded shuffle game $v_{i, j, k, l}^{e}, k, l \in N \backslash\{i, j\}$, belongs to the vector subspace $D_{N}$.

Proof. Section (c) in the above lemma proves that the MLE of an expanded shuffle game $v_{i, j, k, l}^{e}, k, l \in N \backslash\{i, j\}$, in $G_{N}$ with $|N| \geq 5$ agrees with the MLE of the shuffle game $v_{i, j, k, l}$ in a space of cooperative games with only four players, $\{i, j, k, l\}$.

In order to demonstrate that the game $v_{i, j, k, l}^{e}, k, l \in N \backslash\{i, j\}$, is inseparable by modified semivalues, we can consider that the players $i, j, k, l$ are distributed in different coalition blocks in the same way that in the proof of Proposition 3.4. The remaining players $N \backslash\{i, j, k, l\}$ will be distributed in the different blocks next to the players $i, j, k, l$ or they will form new coalition blocks.

Since the variables that correspond to the players in $N \backslash\{i, j, k, l\}$ does not appear in the MLE of the game $v_{i, j, k, l}^{e}$, when we compute allocations for the players $i, j, k, l$ by means of a product of matrices as in (2.5), we obtain the same result as in Proposition 3.4, that is, $\psi_{p}\left[v_{i, j, k, l}^{e}, B\right]=0$ for $p=i, j, k, l, \forall \psi \in \operatorname{Sem}\left(G_{N}\right)$, $\forall B$ coalition structure in $\mathrm{N}$.

For the remaining players, $\psi_{q}\left[v_{i, j, k, l}^{e}, B\right]=0 \forall q \in N \backslash\{i, j, k, l\}$, since the variable $x_{q}$ does not appear in the MLE. 
TABLE 1. Dimensions of the shared kernels $(2 \leq|N| \leq 8)$.

\begin{tabular}{cccccccc}
\hline Cardinality of $N$ & 2 & 3 & 4 & 5 & 6 & 7 & 8 \\
\hline $\operatorname{dim} G_{N}$ & 3 & 7 & 15 & 31 & 63 & 127 & 255 \\
$\operatorname{dim} C_{N}$ & 0 & 0 & 2 & 10 & 32 & 84 & 198 \\
$\operatorname{dim} D_{N}$ & 0 & 0 & 2 & 5 & 9 & 14 & 20 \\
\hline
\end{tabular}

Theorem 5.4. Let $G_{N}$ be a vector space of cooperative TU games with five or more players, $|N|=n \geq 5$. Then,

(a) $\operatorname{dim} D_{N}=\left(\begin{array}{l}n \\ 2\end{array}\right)-n$;

(b) the vector subspace $D_{N}$ is spanned by expanded shuffle games.

Proof. We can see in [1] that the shared kernel $C_{N}$ for $|N| \geq 4$ is spanned by $2^{n}-n^{2}+n-2$ shuffle games whose coalitions with non-null value vary from size $s=2$ to size $s=n-2$. We choose the $\left(\begin{array}{l}n \\ 2\end{array}\right)-n$ shuffle games with coalition size 2. Since they are linearly independent in $G_{N}$, its expanded games are also linearly independent and, by above Proposition, inseparable from the null game by modified semivalues. The linear subspace spanned by these expanded shuffle games is contained in the subspace $D_{N}$ for $|N| \geq 5$.

In addition, because $D_{N} \subseteq C_{N}$, the freedom degrees in $C_{N}$ by a consequence of conditions (2.7) for coalitions with sizes $s>n / 2$ disappear according to the necessary condition of inseparability from the null game in $D_{N}: v(S)=v(N \backslash S)$ (Prop. 4.1). Also, the freedom degrees for coalitions with size from $s=3$ to $s=n / 2$ disappear according to the necessary condition $v(S)=\sum_{T \subset S,|T|=2} v(T)$ $\forall S \subseteq N$ with $3 \leq|S| \leq n / 2$ (Prop. 4.2).

Only the $\left(\begin{array}{l}n \\ 2\end{array}\right)-n$ freedom degrees for coalition size $s=2$ in $C_{N}$ remain in the vector subspace $D_{N}$. Then, the vector subspace spanned by the $\left(\begin{array}{l}n \\ 2\end{array}\right)-n$ expanded shuffle games agrees with $D_{N}$.

\section{Concluding remark}

It is known that every cooperative game with two or three players is separable from the null game by semivalues, i.e., $\operatorname{dim} C_{N}=0$ in cases $|N|=2,3$. Consequently, the vector subspace $D_{N}$ only contains the null game if $|N|=2,3$. For games with four players, Proposition 3.4 proves that both separability concepts coincide: $D_{N}=C_{N}$ for $|N|=4$.

For games with five or more players, the introduction of semivalues modified for games with coalition structure allows us to reduce in a significant way the dimension of the vector subspace of games inseparable from the null game.

The difference of dimensions between the vector subspaces $C_{N}$ and $D_{N}$ shows us how much wider the family of modified semivalues is than the family of semivalues, according to the ability to separate games from the null game. By linearity, the separability between two games is reduced by both solution concepts to separability of their difference from the null game. The ability of separation by semivalues has remarkably increased by the introduction of a priori coalition structures. 


\section{REFERENCES}

[1] R. Amer, J. Derks and J.M. Giménez, On cooperative games, inseparable by semivalues. Int. J. Game Theory 32 (2003) 181-188.

[2] R. Amer and J.M. Giménez, Modification of semivalues for games with coalition structures. Theory Decis. 54 (2003) 185-205.

[3] J.F. Banzhaf, Weighted voting doesn't work: A mathematical analysis. Rutgers Law Rev. 19 (1965) 317-343.

[4] I. Dragan, Potential and consistency for semivalues of finite cooperative TU games. Int. J. Math. Game Theory Algebra 9 (1999) 85-97.

[5] P. Dubey, A. Neyman and R.J. Weber, Value theory without efficiency. Math. Oper. Res. 6 (1981) $122-128$.

[6] G. Owen, Multilinear extensions of games. Manage. Sci. 18 (1972) 64-79.

[7] G. Owen, Multilinear extensions and the Banzhaf value. Naval Res. Log. Quart. 22 (1975) $741-750$.

[8] G. Owen, Values of games with a priori unions, in Essays in Mathematical Economics and Game Theory, edited by R. Henn and O. Moeschelin, Springer-Verlag (1977) 76-88.

[9] G. Owen, Modification of the Banzhaf-Coleman index for games with a priori unions, in Power, Voting and Voting Power, edited by M.J. Holler, Physica-Verlag (1981) 232-238.

[10] L.S. Shapley, A value for n-person games, in Contributions to the Theory of Games II, edited by H.W. Kuhn and A.W. Tucker, Princeton University Press (1953) 307-317.

[11] R.J. Weber, Probabilistic values for games, in The Shapley value: Essays in honor of L.S. Shapley, edited by A.E. Roth, Cambridge University Press (1988) 101-119. 